\title{
Effect of hot water dips on the quality of fresh-cut Ryan Sun peaches
}

\author{
Efecto de las inmersiones en agua caliente sobre la calidad \\ de los duraznos Ryan Sun procesados en fresco
}

\author{
Javier M. Obando-Ulloa ${ }^{1}$, Vanesca Jiménez $z^{2}$,Alejandra Machuca-Vargas ${ }^{2}$,
} John C. Beaulieu ${ }^{3}$, Rodrigo Infante ${ }^{4}$, Víctor H. Escalona-Contreras ${ }^{2 *}$

\begin{abstract}
Fresh-cut products are an important developing food product category, and as a response to current lifestyles they are becoming increasingly popular due to their convenience, nutritious and fresh-like quality. However, fresh-cut produce has limited shelf life because preparation involves physically injuring the tissue, producing subsequent physiological, physical and chemical responses (increasing respiration rate and ethylene production, flavor loss, cut surface discoloration, browning, color loss, decay, increased rate of vitamin loss, rapid softening, shrinkage and shorter storage life), which influences consumer acceptability. The aim of this study was to analyze the effect of hot water dip on the quality of fresh-cut Ryan Sun peaches, applied before or after processing. Fresh-cut Ryan Sun peaches were packaged and stored $6 \mathrm{~d}$ at $5{ }^{\circ} \mathrm{C}$. Respiration rate, firmness, $\mathrm{pH}$, titratable acidy, total phenol content, antioxidant activity and sensory quality were assessed throughout storage. The results demonstrated the efficiency of hot water dip at $50{ }^{\circ} \mathrm{C}$ for 3 min before fresh-cut processing on Ryan Sun peaches to maintain the firmness, lightness and hue angle of the flesh color. This was likely a result of the inactivation of enzymes related to browning and softening. In addition, this treatment allowed the fresh-cut peaches to have the highest TA, which positively influenced the product flavor and thus the acceptability of consumers.
\end{abstract}

Key words: antioxidant capacity, browning, PPO, respiration, total phenols.

\section{RESUMEN}

Los productos cortados en fresco son una importante categoría en desarrollo dentro de la industria de alimentos y como respuesta a los estilos de vida actuales se han popularizado debido a su conveniencia, frescura y calidad nutricional. Sin embargo, los productos cortados en fresco tienen una limitada vida útil debido a que su elaboración daña físicamente el tejido, produciendo una respuesta fisiológica, física y química (aumento de la tasa de respiración y producción de etileno, pérdida de sabor, decoloración de la superficie de corte, pardeamiento, pérdida de color, pudriciones, incremento de la tasa de pérdida de vitaminas, ablandamiento acelerado, marchitez y reducción de la vida de almacenamiento), lo que influye en la aceptación por parte del consumidor. El objetivo de este trabajo consistió en analizar el efecto de las inmersiones en agua caliente sobre la calidad de los duraznos Ryan Sun cortados en fresco, aplicadas antes o después del procesamiento. Los duraznos Ryan Sun cortados en fresco se envasaron y se almacenaron 6 días a $5^{\circ} \mathrm{C}$. Durante el período de almacenamiento se analizaron la tasa de respiración, firmeza, acidez titulable, contenido de compuestos fenólicos totales, actividad antioxidante y calidad sensorial. Los resultados demostraron que las inmersiones en agua caliente a $50{ }^{\circ} \mathrm{C}$ durante $3 \mathrm{~min}$, previo al procesamiento en fresco, son eficientes para mantener la firmeza, la luminosidad y el tono de la pulpa de los duraznos Ryan Sun cortados en fresco, debido a la inactivación de las enzimas relacionadas con el pardeamiento y el ablandamiento. Además, este tratamiento permitió mantener una alta acidez titulable, la que influye positivamente en el sabor del producto y por ende en la aceptabilidad por parte del consumidor.

Palabras clave: capacidad antioxidante, pardeamiento, PPO, respiración, compuestos fenólicos totales.

\section{Introduction}

Fresh-cut products are emerging in the food industry and are becoming increasingly popular due to their convenience, nutritious and fresh-like quality as a response of current lifestyles. The fresh-cut produce industry has experienced an increased growth rate in response to high demand

1 Department of Agroindustry and Enology. Faculty of Agriculture Science. University of Chile. Santa Rosa Ave. 11315, P.O. Box 1004. Santiago, Chile.

2 Center of Postharvest Studies. Faculty of Agriculture Science. University of Chile.

3 United States Department of Agriculture, Agricultural Research Service, Southern Regional Research Center, 1100 Robert E. Lee Boulevard, New Orleans, LA 70124.

4 Department of Plant Production. Faculty of Agriculture Science.University of Chile.

Fecha de Recepción: 5 Noviembre, 2014.

Fecha de Aceptación: 29 Diciembre, 2014. 
by consumers. Nowadays these products are found not only in supermarkets but also in food service distribution chains (Barrett et al., 2010; Beaulieu, 2011; Beaulieu and Gorny, 2004). However, fresh-cut produce has limited shelf life since its preparation necessarily involves physically injuring the tissue (Beaulieu and Gorny, 2004; Oms-Oliu et al., 2010). This unavoidable trauma causes both an immediate increase in the respiration rate and wound-induced ethylene production, and a subsequent physical and chemical response (flavor loss, cut surface discoloration, browning, color loss, decay, increased rate of vitamin loss, rapid softening, shrinkage and shorter storage life) that alters the quality of the product (Beaulieu, 2011; Beaulieu and Gorny, 2004; Loaiza-Velarde and Saltveit, 2001; Salveit, 2000; Siddiq et al., 2013; Steiner et al., 2006).

Consumers judge the quality of fresh-cut fruit and vegetables on the basis of appearance and freshness at the time of purchase (Beaulieu, 2011; Beaulieu and Gorny, 2004; Koukounaras et al, 2008; Oms-Oliu et al., 2010); cut surface discoloration or enzymatic browning, caused by formation of quinones in the presence of $\mathrm{O}_{2}$ and polyphenol oxidase (PPO), is one of the most limiting factors and has been the subject of much research on fresh-cut fruit (Beaulieu and Gorny, 2004; Saltveit, 2000).

Enzymatic browning along with excessive flesh softening and pit cavity breakdown are the major causes of quality deterioration in peach slices as a result of the wounding stress that is associated with changes in color, flavor, aroma and texture (Barrett et al., 2010; Koukounaras et al, 2008; Steiner et al., 2006; Loaiza-Velarde and Saltveit, 2001). Traditionally, browning can be effectively inhibited and prevented by sulfites. However, their use on fresh-cut fruit and vegetables was banned in 1986 by the U.S. Food and Drug Administration (FDA) owing to their potential hazards of allergic-like reactions to the health of sulfite-sensitive asthmatics (Oms-Oliu et al., 2010). Moreover, even though some chemical treatments have proven effective, consumers demand fresh-cut products that maintain fresh characteristics without the use of preservatives, thus the search for alternative browning inhibitors has stimulated considerable research; most GRAS applications used today involve chlorine, ascorbic acid and/or calcium salts for preservation (Beaulieu and Gorny, 2004; Koukounaras et al, 2008; OmsOliu et al., 2010; Saltveit, 2000).
Brief abiotic shocks such as hot water dips, hot water brushing, hot air treatments (vapor heat and forced air) and infrared surface application have emerged as potential alternatives to chemical treatment to affect product physiology; they have shown profound effects on the metabolism of tissues and maintaining the quality of fresh-cut produce (Koukounaras et al, 2008; Lurie, 2006; Saltveit, 2001 and 2000; Siddiq et al., 2013). These treatments are known to interfere with 'normal' protein synthesis by preferentially inducing the synthesis of a unique set of proteins called heat shock proteins that can impact fruit and vegetable quality, affecting tissue respiration, hormone production (particularly ethylene), enzyme activity inhibition, firmness loss, color and visual quality; they have been beneficial in reducing chilling injury and fungus development (Loaiza-Velarde and Saltveit, 2001; Lurie, 2006; Rodríguez et al., 2005; Saltveit, 2005, 2001, 2000; Steiner et al., 2006). However, these treatments have to be adapted to the commodity being treated to achieve the desired effect (pest control, pathogen control or direct effects on the commodity itself) without causing physiological damage (Lurie, 2006).

Hot water treatments can be applied before or after processing. In the former case, the purpose is to affect ripening or senescence processes so that these processes will be delayed in the fresh-cut commodity. However, the effectiveness of these treatments on quality preservation is influenced by species, cultivar, harvest maturity, growing conditions and handling between harvest and treatment (Lurie, 2006; Saltveit, 2001 and 2000). Hot water treatments after cutting have been tested with good results on fresh-cut pears, tomatoes, cantaloupes, Chinese water chestnut, lettuce, celery, cucumber seedling radicles, rice seedlings and peaches (Kang and Saltveit, 2001; Koukounaras et al, 2008; LoaizaVelarde et al., 2003; Loaiza-Velarde and Saltveit, 2001; Lurie, 2006).

However, most published studies testing sanitary treatments have understandably been focused on establishing effective treatment levels and microbial analysis, while sensory appraisals are generally lacking (Beaulieu et al., 2011). In recent years there has been growing dissatisfaction among consumers about the flavor of whole fruits and vegetables, because their sensory properties [color and appearance, flavor (taste and aroma) and texture] which determine food quality and influence the preferences of consumers do not fit the requirements they expect (Baldwin, 
2002). Fresh-cut fruits and vegetables must also have an attractive appearance, acceptable flavor and an appropriate texture to attract initial and continued purchases by consumers (Barrett et al., 2010; Beaulieu, 2011). Therefore, the aim of this study was to evaluate the effects of hot water treatment, applied before or after processing, and the treatment duration on the quality of fresh-cut Ryan Sun peaches stored $6 \mathrm{~d}$ at $5{ }^{\circ} \mathrm{C}$.

\section{Material and Methods}

\section{Plant material}

Ryan Sun peaches were provided free of defects by Univiveros, S.A. (Paine, Región Metropolitana, Chile). The peaches were transported to the laboratory of the Center of Postharvest Studies (CEPOC) at the Faculty of Agricultural Sciences, University of Chile, where fruit were stored at $0 \pm 1{ }^{\circ} \mathrm{C}$ in the cool rooms of CEPOC until processing; 24 fruit were selected for fruit characterization at harvest.

\section{Minimal processing of Ryan Sun peaches}

Fruit was submitted to minimal processing when they reached firmness around $4.9 \mathrm{kgf}$ (Gorny et al., 1998). Minimal processing was conducted at low temperature $\left(5-10^{\circ} \mathrm{C}\right)$ in order to minimize respiration and also to delay deterioration processes such as browning and softening (Steiner et al., 2006). Once the fruit were peeled and cored, they were dipped for $5 \mathrm{~min}$ in cold water $\left(5^{\circ} \mathrm{C}\right)$ containing $200 \mathrm{mg} \mathrm{L}^{-1}$ $\mathrm{NaOCl}$. Subsequently each fruit was cut with a sharp stainless steel knife into 10 wedges (mean mass $20 \mathrm{~g}$ and $2 \mathrm{~cm}$ thickness), which were also dipped for 5 min in cold water $\left(5^{\circ} \mathrm{C}\right)$ containing $100 \mathrm{mg}^{-1} \mathrm{NaOCl}$ and then rinsed for $5 \mathrm{~min}$ with tap water at $5^{\circ} \mathrm{C}$.

\section{Hot water dip treatment duration}

In order to evaluate the effectiveness of applying hot water dip at $50{ }^{\circ} \mathrm{C}$ before or after fresh-cut processing to prolong the shelf life, whole peaches were dipped for 0,3 or 6 min, while fresh-cut Ryan Sun peaches were treated for 0,1 or $3 \mathrm{~min}$.

\section{Packaging}

Approximately $200 \mathrm{~g}$ (10 wedges) of fresh-cut peach were placed in $500 \mathrm{~mL}$ polyethylene trays
(Ultrapac Sudamericana, Santiago, Chile). The trays were sealed with a polyvinyl chloride film (Europlast, Santiago, Chile) and were stored $6 \mathrm{~d}$ at $5{ }^{\circ} \mathrm{C}$.

\section{Analysis of fresh-cut fruit quality}

Three trays of each treatment were randomly selected and removed from cold storage after 1, 3 and $6 \mathrm{~d}$. Color, firmness, soluble solids content, $\mathrm{pH}$, titratable acidity, total phenol content, antioxidant capacity and sensory analysis were evaluated.

\section{Respiration rate}

Immediately after cutting, approximately $500 \mathrm{~g}$ of fresh-cut peaches were placed in glass jars $(0.5 \mathrm{~L})$ fitted with air-tight lids equipped with a rubber septum. Jars were stored at $5{ }^{\circ} \mathrm{C}$ to monitor the respiration rate of fresh-cut peaches during storage. In each daily analysis, jars were closed for at least $3 \mathrm{~h}$. Then a $10 \mathrm{~mL}$ gas sample was taken from the headspace of every jar with a syringe (Nitro, Argentina), which was injected into a gas chromatograph (GC) HP 5890 series II (Hewlett Packard Co., Rockville, Md., U.S.A.) fitted with a Hayesep Q column $(2.4 \mathrm{~m} \times 3 \mathrm{~mm})$ and a thermal conductivity detector (TCD). Injector and oven temperatures were set at $50{ }^{\circ} \mathrm{C}$, while the detector was at $200^{\circ} \mathrm{C}$. Helium was used as the carrier gas at 50 psi (Fernández-Trujillo and Artés, 1997). The GC was calibrated every day with a $5 \% \mathrm{O}_{2}$ and $10 \%$ $\mathrm{CO}_{2}$ standard (Indura, Santiago, Chile). Respiration rate was expressed in $\mathrm{mg} \mathrm{CO} \cdot \mathrm{kg}^{-1} \cdot \mathrm{h}^{-1}$.

\section{Color}

Fresh-cut peach color $\left(L^{*}, a^{*}, b^{*}\right)$ was determined on the inner (flesh) and outer (peel) surfaces of all the slices contained in every package with a Minolta chroma meter (model CR 300, Tokyo, Japan). The chroma meter was calibrated daily with the white tile provided with the equipment. The color values of $a^{*}$ and $b^{*}$ were further converted into hue angle $\left[\right.$ hue $\left.=\tan ^{-1}(b / a)\right]$ and chroma $\left[\right.$ chroma $=\left(a^{2}\right.$ $\left.+b^{2}\right)^{0.5}$, according to McGuire (1992).

\section{Texture}

The firmness of fresh-cut peaches was determined using a Fruit Texture Analyzer (FTA, TR, Forli, Italy). Compression firmness was measured with a 
7.9-mm-diameter cylindrical probe to a deformation of $10 \mathrm{~mm}$ at $30 \mathrm{~mm} \cdot \mathrm{s}^{-1}$. The peak force was recorded and used to indicate firmness of the fresh-cut product.

\section{Soluble solid content, $\mathrm{pH}$ and titratable acidity (TA)}

These parameters were determined with a representative sample of juice obtained with an electric juicer (Juice Factory, Black\&Decker, U.S.A.) from the slices of the trays of fresh-cut peaches. Soluble solid content (SSC), expressed as ${ }^{\circ} \mathrm{Brix}$, was measured on a representative sample of juice from fresh-cut peaches using a temperaturecompensated ATC-1 refractometer (Atago, Tokyo, Japan). pH was measured with a pH meter (Schott, Germany). The titratable acidity was determined in $10 \mathrm{~mL}$ of juice by titrating with $0.1 \mathrm{~N} \mathrm{NaOH}$ to $\mathrm{pH}$ 8.2-8.3, and expressed as percentage of malic acid (Fernández-Trujillo et al., 2005).

\section{Analysis of total phenol content and antioxidant capacity}

For the analysis of total phenol content and antioxidant capacity, the flesh of fresh-cut peach samples was previously frozen with liquid nitrogen and stored at $-20^{\circ} \mathrm{C}$ until analysis.

\section{Total phenol content}

The methodology for total phenol quantification was based on Velioglu et al. (1998). Twenty g of fresh-cut peach flesh was blended with $50 \mathrm{~mL} 80 \%$ methanol for $2 \mathrm{~min}$ and subsequently homogenized with a LabTech stirred shaker (LMS-1003, Daihan Labtech Co. Ltd., Korea) for $1 \mathrm{~h}$, then it was centrifuged (Heraeus, Labofuge 200, Germany) at 5300 rpm for 40 min and the supernatant was vacuum filtered through a $0.45 \mu \mathrm{m}$ membrane (Millipore, Brazil). The pellet was extracted again with $80 \%$ methanol. The supernatants were combined and a $1 \mathrm{~mL}$ aliquot was diluted in $20 \mathrm{~mL}$ distilled water; absorbance was read at 280 $\mathrm{nm}$ with a Shimadzu spectrophotometer (UV-1700, Japan). The results were expressed as milligrams of gallic acid equivalent (GAE) per gram fresh weight.

\section{Antioxidant activity}

The 2,2-diphenyl-1-picrylhydrazyl (DPPH•) method described by Brand-Williams et al. (1995) was used to determine the antioxidant activity of fresh-cut peach. Flesh from the fresh-cut peach was homogenized (Ultra-Turrax IKA, T18 Basic, Germany) with $20 \mathrm{~mL}$ of methanol: water solution (80:20, $200 \mu \mathrm{L} \mathrm{HCl})$ at $200 \mathrm{rpm}$ for $1 \mathrm{~h}$ min. Then the solution was centrifuged $5 \mathrm{~min}$ at $3500 \mathrm{rpm}$. The supernatant was filtered through a Millipore membrane and serial solutions were prepared with methanol; $0.1 \mathrm{~mL}$ of this solution was mixed with 3.9 $\mathrm{mL} \mathrm{f} \mathrm{DPPH} \cdot(20 \mathrm{ppm})$ methanolic solution and kept in the dark for $30 \mathrm{~min}$ at $23.5 \pm 1^{\circ} \mathrm{C}$; the decrease in the absorbance was measured at $517 \mathrm{~nm}$ in the Shimadzu spectrophotometer. DPPH • activity was expressed as milligrams of ascorbic acid equivalent antioxidant capacity per $\mathrm{g}$ fresh weight.

\section{Sensory evaluation}

An in-house trained sensory panel of 12 judges was used to evaluate the quality attributes of freshcut peaches. Two peach wedges were served to each panelist on dishes labeled with a 3-digit code using a completely randomized order. The analysis was conducted in individual temperature-controlled tasting booths in the sensory evaluation laboratory. The judges were required to cleanse their palates with a sip of water at room temperature. The sensory attributes in terms of aroma, sweetness, sourness, juiciness, texture, flavor, flesh browning and acceptability were evaluated on an unstructured continuous 150-mm scale graded from low to high. The data were collected on a paper ballot.

\section{Statistical analysis}

Data were submitted to ANOVA using JMP v8.0 for Windows (SAS Institute Inc., NC). If significant differences at $p<0.05$ were identified, data were analyzed as a 2-factor linear model using JMP v8.0 for Windows (SAS Institute Inc., $\mathrm{NC}$ ) with treatment $(\mathrm{T})$ and storage time $(\mathrm{t})$ as the factors. Sensory analysis data was also submitted to a principal components analysis (PCA) in JMP. This analysis was used to identify the sensory parameters that explain the most variance in differences among treatments and to group the treatments according to the judges' perception. The PCA graph was split into two plots (score and loading plots, respectively) to facilitate visualization of the analysis. The score plot was performed with the mean component value obtained for each PC, while the loading plot used 
the eigenvectors greater than 0.20 for the sensory parameters that better define the orientation of each PC axis. The first principal component (PC1) represents the greatest amount of variation resulting from a combination of all of the variables. The second principal component (PC2) represents the next greatest source of variation, and so on (Obando et al., 2008; Obando-Ulloa et al., 2008). Pearson product moment correlation coefficients found in the principal components analysis (PCA) for sensory analysis data were interpreted empirically: the correlation between two sensory attributes was considered to be 'weak' if $r=0-0.3$, 'moderate' if $r=$ 0.31-0.7 and 'high' if $r=0.71-1.0$ (Fan et al., 2010).

\section{Results}

\section{Characterization of fruit at harvest}

At harvest, 'Ryan Sun' peach showed a fruit weight of $257.6 \pm 25.3 \mathrm{~g}$ and approx. $11.1 \pm 1.2$ ${ }^{\circ}$ Brix (Table 1). Peel color was characterized by a $\mathrm{L}^{*}, \mathrm{C}^{*}$ and ${ }^{\circ}$ hue of 55.3, 29.4 and 56.6, respectively. In addition, 'Ryan Sun' peach at harvest had fruit firmness around $6.2 \mathrm{kgf}$, and it decreased to $4.9 \mathrm{kgf}$ before processing. Whole fruit at harvest also had a total phenols content of $0.54 \mathrm{mg} \mathrm{EAG} \cdot \mathrm{mg}_{\mathrm{FW}}{ }^{-1}$ and an antioxidant capacity of $1.2 \mathrm{~g}_{\mathrm{FW}} \cdot \mathrm{mg}_{\mathrm{DPPH}}{ }^{-1}$.

\section{Respiration rate}

All fresh-cut treatments had a respiration rate approx. 24\% higher than the whole fruit $(21.5$ $\left.\mathrm{mg} \mathrm{CO} \cdot \mathrm{kg}^{-1} \cdot \mathrm{h}^{-1}\right)$ at the beginning of storage, but statistical differences were not found $(P>0.05)$ among treatments during storage at $5{ }^{\circ} \mathrm{C}$ (Figura 1$)$.

Table 1. Physical and chemical quality parameters of 'Ryan Sun' peach at harvest

\begin{tabular}{lrr}
\hline Quality parameter & \multicolumn{2}{c}{ Mean \pm SD } \\
\hline Weight $(\mathrm{g})$ & $257.57 \pm$ & 25.33 \\
$\mathrm{SSC}\left({ }^{\circ} \mathrm{Brix}\right)$ & $11.05 \pm$ & 1.18 \\
$\mathrm{~L}^{*}$ & $55.29 \pm$ & 5.75 \\
$\mathrm{a}^{*}$ & $15.54 \pm$ & 6.40 \\
$\mathrm{~b}^{*}$ & $23.93 \pm$ & 2.92 \\
$\mathrm{C}^{*}$ & $29.37 \pm$ & 2.21 \\
${ }^{o}$ hue & $56.57 \pm$ & 13.79 \\
Fruit firmness $(\mathrm{N})$ & $6.22 \pm$ & 0.98 \\
Total phenols content $\left(\mathrm{mg} \mathrm{GAE} \cdot \mathrm{g}_{\mathrm{fw}}{ }^{-1}\right)$ & $0.54 \pm$ & 0.02 \\
Antioxidant capacity $\left(\mathrm{g}_{\mathrm{fg}} \cdot \mathrm{mg}_{\mathrm{DPPH}}{ }^{-1}\right)$ & $1.20 \pm$ & 0.10 \\
\hline
\end{tabular}

At the end of storage (6d), the respiration rate had increased by around $15 \%\left(34.05 \mathrm{mg} \mathrm{CO} \cdot \mathrm{kg}^{-1} \cdot \mathrm{h}^{-1}\right)$, but no differences $(P>0.05)$ were found among treatments.

\section{Color}

Lightness ( $\left.\mathrm{L}^{*}\right)$ increased from 56 to $60 \%$ as an effect of time $(p<0.0001$; Table 2$)$. In addition, it was also affected by treatment $(p<0.0001)$. Those fruit submitted to fresh-cut processing before water dip at 5 and $50{ }^{\circ} \mathrm{C}$ for 1 and 3 min displayed the lowest $\mathrm{L}^{*}$ values (darker color), while the highest lightness (lightest color) was found in the whole fruit treated $3 \mathrm{~min}$ in water at $50^{\circ} \mathrm{C}$ and then submitted to fresh-cut processing (Table 2). On the other hand, fruit submitted to fresh-cut processing before dip either in water at $5{ }^{\circ} \mathrm{C}(1 \mathrm{~min})$ or hot water dip at $50{ }^{\circ} \mathrm{C}$ for 3 or $6 \mathrm{~min}$ displayed a more vivid color (higher $\mathrm{C}^{*}$ value $~ 30 \%$ ) than those submitted to water dip at 5 or $50{ }^{\circ} \mathrm{C}$ before fresh-cut processing $(P<0.0001$; Table 2).

Hue angle was the only color parameter which was affected by the interaction of $\mathrm{T} \times \mathrm{t}(p<0.05)$. The flesh of whole peaches previously dipped $3 \mathrm{~min}$ in hot water $50^{\circ} \mathrm{C}$ and then submitted to fresh-cut processing had a darker yellow color (higher $\mathrm{H}^{*}$ ) after $4 \mathrm{~d}$ at $5{ }^{\circ} \mathrm{C}$ among all treatments, while freshcut peaches submitted $1 \mathrm{~min}$ to hot water at $50^{\circ} \mathrm{C}$ were the most affected since they showed a lower yellow color (lower $\mathrm{H}^{*}$ ) after $1 \mathrm{~d}$ at $5^{\circ} \mathrm{C}$ than other treatments. There was an increasing trend in the yellow color of the fresh-cut peaches treated with water dip either before or after processing (Table 2). This trend may be interpreted as the hot water dip

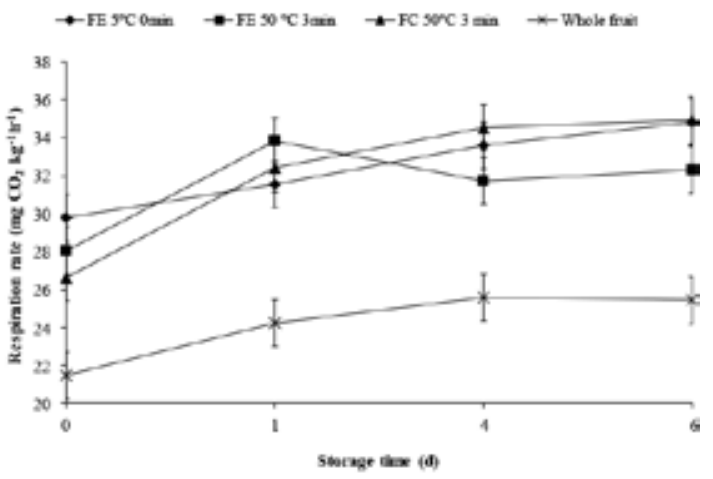

Figure 1. Respiration rate evolution (mean \pm SE, $n=3$ ) of fresh-cut Ryan Sun peaches submitted to hot water dip before or after processing and stored $6 \mathrm{~d}$ at $5^{\circ} \mathrm{C}$. 
Table 2. Flesh color of fresh cut 'Ryan Sun' peaches submitted to hot water dips before or after processing and stored $6 \mathrm{~d}$ at $5{ }^{\circ} \mathrm{C}$.

\begin{tabular}{|c|c|c|c|c|c|c|c|c|}
\hline \multirow{2}{*}{ Color parameter } & \multirow{2}{*}{\multicolumn{2}{|c|}{ Treatment }} & \multicolumn{5}{|c|}{ Shelf life (d) } & \multirow{2}{*}{ Mean } \\
\hline & & & 1 & & 4 & 6 & 6 & \\
\hline \multirow[t]{7}{*}{$\mathrm{L}^{*}$} & \multicolumn{2}{|c|}{$\mathrm{WD} 5^{\circ} \mathrm{C} 1 \mathrm{~min}+\mathrm{FCP}$} & 56.4 & 60.0 & & 61.2 & & $59.7 \mathrm{ab}$ \\
\hline & \multicolumn{2}{|c|}{ WD $50^{\circ} \mathrm{C} 3 \mathrm{~min}+\mathrm{FCP}$} & 61.3 & 64.0 & & 63.8 & & $63.2 \mathrm{a}$ \\
\hline & \multicolumn{2}{|c|}{$\mathrm{WD} 50^{\circ} \mathrm{C} 6 \mathrm{~min}+\mathrm{FCP}$} & 59.3 & 62.3 & & 64.3 & & $62.6 \mathrm{ab}$ \\
\hline & \multicolumn{2}{|c|}{$\mathrm{FCP}+\mathrm{WD} 5^{\circ} \mathrm{C}, 1 \mathrm{~min}$} & 59.7 & 54.1 & & 61.4 & & $59.1 \mathrm{~b}$ \\
\hline & \multicolumn{2}{|c|}{$\mathrm{FCP}+\mathrm{WD} 50^{\circ} \mathrm{C}, 1 \mathrm{~min}$} & 51.7 & 50.6 & & 57.5 & & $54.3 \mathrm{c}$ \\
\hline & \multicolumn{2}{|c|}{$\mathrm{FCP}+\mathrm{WD} 50^{\circ} \mathrm{C}, 3 \mathrm{~min}$} & 52.4 & 51.4 & & 56.1 & & $54.0 \mathrm{c}$ \\
\hline & \multicolumn{2}{|l|}{ Mean } & 56.8 B & 57.1 & B & 60.7 & $\mathbf{A}$ & \\
\hline \multirow[t]{6}{*}{$\mathrm{C}^{*}$} & \multicolumn{2}{|c|}{$\mathrm{WD} 5^{\circ} \mathrm{C} 1 \mathrm{~min}+\mathrm{FCP}$} & 27.5 & 25.8 & & 28.1 & & $27.4 \mathrm{~b}$ \\
\hline & \multicolumn{2}{|c|}{ WD $50^{\circ} \mathrm{C} 3 \mathrm{~min}+\mathrm{FCP}$} & 30.2 & 27.0 & & 28.7 & & $28.6 \mathrm{ab}$ \\
\hline & \multicolumn{2}{|c|}{$\mathrm{WD} 50^{\circ} \mathrm{C} 6 \mathrm{~min}+\mathrm{FCP}$} & 27.1 & 27.3 & & 28.1 & & $27.6 \mathrm{~b}$ \\
\hline & \multicolumn{2}{|c|}{$\mathrm{FCP}+\mathrm{WD} 5^{\circ} \mathrm{C}, 1 \mathrm{~min}$} & 28.5 & 29.6 & & 29.1 & & $29.1 \mathrm{a}$ \\
\hline & \multicolumn{2}{|c|}{$\mathrm{FCP}+\mathrm{WD} 50^{\circ} \mathrm{C}, 1 \mathrm{~min}$} & 29.9 & 30.0 & & 30.0 & & $30.0 \mathrm{a}$ \\
\hline & \multicolumn{2}{|c|}{$\mathrm{FCP}+\mathrm{WD} 50^{\circ} \mathrm{C}, 3 \mathrm{~min}$} & 30.2 & 30.2 & & 29.6 & & 29.9 a \\
\hline \multirow[t]{6}{*}{$\mathrm{h}^{\mathrm{o}}$} & \multicolumn{2}{|c|}{$\mathrm{WD} 5^{\circ} \mathrm{C} 1 \mathrm{~min}+\mathrm{FCP}$} & $72.2 \mathrm{~g}, \mathrm{~h}, \mathrm{i}$ & 76.8 & $\mathrm{c}, \mathrm{d}, \mathrm{e}$ & 76.7 & $\mathrm{c}, \mathrm{d}, \mathrm{e}$ & \\
\hline & \multicolumn{2}{|c|}{$\mathrm{WD} 50^{\circ} \mathrm{C} 3 \mathrm{~min}+\mathrm{FCP}$} & $79.2 \mathrm{a}, \mathrm{b}, \mathrm{c}$ & 81.6 & $\mathrm{a}$ & 79.7 & $\mathrm{a}, \mathrm{b}, \mathrm{c}$ & \\
\hline & \multicolumn{2}{|c|}{$\mathrm{WD} 50^{\circ} \mathrm{C} 6 \mathrm{~min}+\mathrm{FCP}$} & $75.1 \mathrm{~d}, \mathrm{e}, \mathrm{f}$ & 78.1 & $\mathrm{~b}, \mathrm{c}, \mathrm{d}$ & 81.0 & $\mathrm{a}, \mathrm{b}$ & \\
\hline & \multicolumn{2}{|c|}{$\mathrm{FCP}+\mathrm{WD} 5^{\circ} \mathrm{C}, 1 \mathrm{~min}$} & $76.6 \mathrm{c}, \mathrm{d}, \mathrm{e}$ & 74.5 & $e, f, g$ & 77.4 & $\mathrm{~b}, \mathrm{c}, \mathrm{d}$ & \\
\hline & \multicolumn{2}{|c|}{$\mathrm{FCP}+\mathrm{WD} 50^{\circ} \mathrm{C}, 1 \mathrm{~min}$} & $69.7 \mathrm{i}$ & 74.3 & $\mathrm{e}, \mathrm{f}, \mathrm{g}$ & 75.5 & $\mathrm{c}, \mathrm{d}, \mathrm{e}$ & \\
\hline & \multicolumn{2}{|c|}{$\mathrm{FCP}+\mathrm{WD} 50^{\circ} \mathrm{C}, 3 \mathrm{~min}$} & $72.6 \mathrm{f}, \mathrm{g}, \mathrm{h}$ & 71.2 & $\mathrm{~h}, \mathrm{i}$ & 76.3 & $\mathrm{c}, \mathrm{d}, \mathrm{e}$ & \\
\hline \multirow[t]{4}{*}{ Significance } & \multirow{4}{*}{$\begin{array}{l}\text { Treatment }(\mathrm{T}) \\
\text { Storage }(\mathrm{t}) \\
\mathrm{T} \times \mathrm{t}\end{array}$} & $\mathrm{L}^{*}$ & $\mathrm{C}^{*}$ & \multicolumn{2}{|l|}{$\mathrm{h}^{\mathrm{o}}$} & & & \\
\hline & & $* * * *$ & $* * * *$ & \multicolumn{2}{|l|}{$* * * *$} & & & \\
\hline & & $* * * *$ & NS & $* * * *$ & & & & \\
\hline & & NS & NS & $*$ & & & & \\
\hline
\end{tabular}

Capital letters compare means along storage, while small letters compare means among treatment. Means not connected by same letter are significantly different according to Tuckey's test at $P<0.05$. NS: not significant, $* P<0.05$, ** $P<0.01, * * * P<0.001$, ***** $P<0.0001$.

treatment efficiency to keep the flesh yellow color of the fresh-cut Ryan Sun peaches.

\section{Firmness}

Fruit firmness showed statistical differences $(p<0.05)$ among treatments, due to the interaction $\mathrm{T} \times \mathrm{t}$ (Table 3). Those fruit dipped in hot water at $50^{\circ} \mathrm{C}$ for $3 \mathrm{~min}$ before fresh-cut processing showed the highest firmness $(3.56 \mathrm{kgf})$ after $1 \mathrm{~d}$ at $5^{\circ} \mathrm{C}$, while the lowest firmness was observed in fresh-cut peaches treated $1 \mathrm{~min}$ in water dip at $5^{\circ} \mathrm{C}$ after $4 \mathrm{~d}$ at $5^{\circ} \mathrm{C}$, in comparison to other treatments stored in the same conditions. All treatments displayed a firmness loss ranging from 2 to $28 \%$ during storage, which was more pronounced in those peaches submitted to water dip before processing. An opposite trend was observed in fresh-cut peaches submitted to water dip 3 min at $50{ }^{\circ} \mathrm{C}$ (data not shown).

\section{Soluble solid content, $\mathrm{pH}$ and titratable acidity (TA)}

Fresh-cut peaches showed a mean soluble solid content around $13{ }^{\circ} \mathrm{Brix}$, which was not affected by either treatment $(\mathrm{T})$, storage $(\mathrm{t})$ or the interaction of both factors $(\mathrm{T} \times \mathrm{t})$ (data not shown). $\mathrm{pH}$ generally increased during the storage as an effect of time $(\mathrm{p}<0.05)$. On day 1 the treatments the $\mathrm{pH}$ mean was around 3.94, and at the end of storage $\mathrm{pH}$ was about 4.10 (Table 4). TA showed significant differences as an effect of storage $(\mathrm{t}, p<0.01)$ and treatment $(\mathrm{T}, p<0.0001)$. TA decreased during storage from $0.48 \%$ to $0.40 \%$ (malic acid), while whole peaches previously dipped $6 \mathrm{~min}$ in hot water at $50{ }^{\circ} \mathrm{C}$ and then submitted to fresh-cut processing had the highest TA $(0.44 \%)$ compared to fresh-cut peaches dipped $3 \mathrm{~min}$ in hot water at $50{ }^{\circ} \mathrm{C}$ (Table 5). 
Table 3. Firmness (kgf) of fresh cut 'Ryan Sun' peaches submitted to hot water dips before or after processing and stored $6 \mathrm{~d}$ at $5{ }^{\circ} \mathrm{C}$.

\begin{tabular}{lllc}
\hline \multirow{2}{*}{ Treatment } & \multicolumn{3}{c}{ Storage (d) } \\
\cline { 2 - 4 } & \multicolumn{1}{c}{1} & \multicolumn{1}{c}{6} \\
\hline WF 5 ${ }^{\circ} \mathrm{C} 1 \mathrm{~min}+\mathrm{FCP}$ & $2.69 \mathrm{~b}, \mathrm{c}$ & $2.80 \mathrm{a}, \mathrm{b}$ & $1.94 \mathrm{~b}, \mathrm{c}$ \\
WF 50 ${ }^{\circ} \mathrm{C} 3 \mathrm{~min}+\mathrm{FCP}$ & $3.56 \mathrm{a}$ & $2.80 \mathrm{a}, \mathrm{b}$ & $2.79 \mathrm{a}, \mathrm{b}$ \\
WF 50 ${ }^{\circ} \mathrm{C} 6 \mathrm{~min}+\mathrm{FCP}$ & $2.75 \mathrm{a}, \mathrm{b}$ & $2.31 \mathrm{c}, \mathrm{d}$ & $2.14 \mathrm{~d}, \mathrm{e}$ \\
$\mathrm{FCP}+$ WD 5 ${ }^{\circ} \mathrm{C}, 1 \mathrm{~min}$ & $2.78 \mathrm{a}, \mathrm{b}$ & $1.65 \mathrm{~g}$ & $2.38 \mathrm{c}, \mathrm{d}$ \\
$\mathrm{FCP}+$ WD 50 ${ }^{\circ} \mathrm{C}, 1 \mathrm{~min}$ & $2.32 \mathrm{c}, \mathrm{d}$ & $1.68 \mathrm{f}, \mathrm{g}$ & $1.76 \mathrm{e}, \mathrm{f}$ \\
FCP + WD 50 ${ }^{\circ} \mathrm{C}, 3 \mathrm{~min}$ & $2.68 \mathrm{~b}, \mathrm{c}$ & $2.14 \mathrm{~d}, \mathrm{e}$ & $2.62 \mathrm{~b}, \mathrm{c}$ \\
\hline
\end{tabular}

Significance

$\begin{array}{rc}\text { Treatment (T) } & \text { NS } \\ \text { Storage }(\mathrm{t}) & \mathrm{NS} \\ \mathrm{T} \times \mathrm{t} & *\end{array}$

Letters compare means among treatment. Means not connected by same letter are significantly different according to Tuckey's test at $P<0.05$.NS: not significant, $* P<0.05$, ** $P<0.01$, *** $P<0.001, * * * * P<0.0001$.

Table 4. $\mathrm{pH}$ of fresh cut 'Ryan Sun' peachessubmitted to hot water dips before or after processing and stored $6 \mathrm{~d}$ at $5{ }^{\circ} \mathrm{C}$.

\begin{tabular}{|c|c|c|c|}
\hline \multirow{2}{*}{ Treatment } & \multicolumn{3}{|c|}{ Storage (d) } \\
\hline & 1 & 4 & 6 \\
\hline $\mathrm{WD} 5^{\circ} \mathrm{C} 1 \mathrm{~min}+\mathrm{FCP}$ & 3.91 & 4.01 & 4.12 \\
\hline $\mathrm{WD} 50^{\circ} \mathrm{C} 3 \mathrm{~min}+\mathrm{FCP}$ & 3.94 & 3.99 & 4.05 \\
\hline $\mathrm{WD} 50^{\circ} \mathrm{C} 6 \mathrm{~min}+\mathrm{FCP}$ & 3.84 & 3.92 & 4.07 \\
\hline $\mathrm{FCP}+\mathrm{WD} 5^{\circ} \mathrm{C}, 1 \mathrm{~min}$ & 3.86 & 4.01 & 4.23 \\
\hline $\mathrm{FCP}+\mathrm{WD} 50^{\circ} \mathrm{C}, 1 \mathrm{~min}$ & 3.79 & 4.05 & 4.14 \\
\hline $\mathrm{FCP}+\mathrm{WD} 50^{\circ} \mathrm{C}, 3 \mathrm{~min}$ & 4.15 & 4.00 & 4.17 \\
\hline Mean & $3.94 \mathrm{~B}$ & 4.00 Ab & $4.13 \mathrm{a}$ \\
\hline \multicolumn{4}{|l|}{ Significance } \\
\hline Treatment $(\mathrm{T})$ & NS & & \\
\hline Storage $(\mathrm{t})$ & $*$ & & \\
\hline $\mathrm{T} \times \mathrm{t}$ & NS & & \\
\hline
\end{tabular}

Letters compare means along storage. Means not connected by same letter are significantly different according to Tuckey's test at $\mathrm{P}<0.05$. NS: not significant, $* \mathrm{P}<0.05$, $* * \mathrm{P}<0.01$, *** $\mathrm{P}$ $<0.001, * * * * \mathrm{P}<0.0001$

\section{Total phenol content}

The raw material at harvest had a total phenol content of $0.54 \mathrm{mg} \mathrm{GAE} \cdot \mathrm{g}_{\mathrm{FW}}{ }^{-1}$ (Table 1). However, this content was mainly affected by $\mathrm{T} \times \mathrm{t}$ interaction $(p<0.0001)$. Fresh-cut peaches submitted $1 \mathrm{~min}$ to water dip at $5{ }^{\circ} \mathrm{C}$ showed the highest total phenol
Table 5. Titratable acidity (\% malic acid) of fresh cult 'Ryan Sun' peaches submitted to hot water dips before or after processing and stored $6 \mathrm{~d}$ at $5{ }^{\circ} \mathrm{C}$.

\begin{tabular}{lcccc}
\hline \multirow{2}{*}{ Treatment } & \multicolumn{3}{c}{ Storage (d) } & \multirow{2}{*}{ Mean } \\
\cline { 2 - 4 } & 1 & 4 & 6 & \\
\hline WD $5{ }^{\circ} \mathrm{C} 1 \mathrm{~min}+\mathrm{FCP}$ & 0.64 & 0.53 & 0.39 & $0.43 \mathrm{ab}$ \\
WD 50 ${ }^{\circ} \mathrm{C} 3 \mathrm{~min}+\mathrm{FCP}$ & 0.45 & 0.37 & 0.46 & $0.42 \mathrm{bc}$ \\
WD 50 ${ }^{\circ} \mathrm{C} 6 \mathrm{~min}+\mathrm{FCP}$ & 0.53 & 0.44 & 0.41 & $0.44 \mathrm{a}$ \\
$\mathrm{FCP}+\mathrm{WD} 5{ }^{\circ} \mathrm{C}, 1 \mathrm{~min}$ & 0.42 & 0.36 & 0.38 & $0.37 \mathrm{bc}$ \\
$\mathrm{FCP}+\mathrm{WD} 50{ }^{\circ} \mathrm{C}, 1 \mathrm{~min}$ & 0.49 & 0.34 & 0.42 & $0.40 \mathrm{abc}$ \\
$\mathrm{FCP}+\mathrm{WD} 50{ }^{\circ} \mathrm{C}, 3 \mathrm{~min}$ & 0.43 & 0.32 & 0.34 & $0.35 \mathrm{c}$ \\
\hline Mean & $\mathbf{0 . 4 8} \mathbf{A}$ & $\mathbf{0 . 3 8} \mathbf{B}$ & $\mathbf{0 . 4 0} \mathbf{~ B}$ \\
\hline Significance & & & & \\
Treatment (T) & $* * * *$ & & & \\
Storage (t) & $* *$ & & & \\
$\mathrm{~T} \times \mathrm{t}$ & $\mathrm{NS}$ & &
\end{tabular}

Capital letters compare means along storage, while small letters compare means among treatments. Means not connected by same letter are significantly different according to Tuckey's test at $P<0.05$. NS: not significant, $* P<0.05$, ** $P<0.01$, $* * * P<0.001$, **** $P<0.0001$.

content $\left(0.82 \mathrm{mg} \mathrm{GAE} \cdot \mathrm{g}_{\mathrm{FW}}{ }^{-1}\right)$, followed by fresh-cut peaches submitted to $3 \mathrm{~min}$ hot water dip at $50^{\circ} \mathrm{C}$ $\left(0.65 \mathrm{mg} \mathrm{GAE} \cdot \mathrm{F}_{\mathrm{FW}}{ }^{-1}\right)$ after $1 \mathrm{~d}$ of storage at $5^{\circ} \mathrm{C}$. The lowest content was observed in those fresh-cut peaches submitted to $1 \mathrm{~min}$ hot water dip at $50{ }^{\circ} \mathrm{C}$ $\left(0.16 \mathrm{mg} \mathrm{GAE} \cdot \mathrm{g}_{\mathrm{FW}}{ }^{-1}\right)$ at the end of storage (Table 6).

\section{Antioxidant activity}

The raw material at harvest had an antioxidant capacity of $1.20 \mathrm{~g}_{\mathrm{FW}} \cdot \mathrm{mg}_{\mathrm{DPPH}}{ }^{-1}$ (Table 1 ). However, this antioxidant capacity was mainly affected by $\mathrm{T} \times$ $\mathrm{t}$ interaction $(p<0.0001)$. Those fresh-cut peaches submitted for $3 \mathrm{~min}$ to water at $50^{\circ} \mathrm{C}$ displayed the highest antioxidant activity $\left(1.24 \mathrm{~g}_{\mathrm{FW}} \cdot \mathrm{mg}_{\mathrm{DPPH}}{ }^{-1}\right)$ at the end of storage at $5^{\circ} \mathrm{C}$ among all the treatments. Meanwhile, the fresh-cut peaches submitted 3 min to hot water dip at $50^{\circ} \mathrm{C}$ showed the lowest antioxidant activity $\left(0.17 \mathrm{~g}_{\mathrm{FW}} \cdot \mathrm{mg}_{\mathrm{DPPH}}{ }^{-1}\right)$ at the end of storage at $5^{\circ} \mathrm{C}$ (Table 7).

\section{Sensory evaluation}

The fresh-cut peach attributes acid taste, juiciness, texture and appearance were not affected by the treatment $(\mathrm{T})$, storage $(\mathrm{t})$ or the interaction of these factors $(\mathrm{T} \times \mathrm{t})$. Storage $(\mathrm{t})$ mainly affected acceptability $(p<0.0001)$, aroma $(p<0.0001)$, 
Table 6. Total phenols content $\left(\mathrm{mg}_{\mathrm{GAE}} \cdot \mathrm{g}_{\mathrm{FW}}{ }^{-1}\right)$ of fresh cut 'Ryan Sun' peaches submitted to hot waterdips before or after processing and stored $6 \mathrm{~d}$ at $5{ }^{\circ} \mathrm{C}$.

\begin{tabular}{|c|c|c|c|c|c|}
\hline \multirow{2}{*}{ Treatment } & \multicolumn{3}{|c|}{ Storage (d) } & \multirow{2}{*}{\multicolumn{2}{|c|}{ Mean }} \\
\hline & 1 & 4 & 6 & & \\
\hline $\mathrm{WD} 5^{\circ} \mathrm{C} 1 \mathrm{~min}+\mathrm{FCP}$ & n.d & n.d & n.d. & n.d & \\
\hline $\mathrm{WD} 50^{\circ} \mathrm{C} 3 \mathrm{~min}+\mathrm{FCP}$ & $0.43 \mathrm{c}, \mathrm{d}$ & $0.35 \mathrm{~d}, \mathrm{e}$ & $0.33 \mathrm{e}, \mathrm{f}$ & 0.37 & c, d \\
\hline $\mathrm{WD} 50^{\circ} \mathrm{C} 6 \mathrm{~min}+\mathrm{FCP}$ & $0.32 \mathrm{e}, \mathrm{f}, \mathrm{g}$ & $0.26 \mathrm{f}, \mathrm{g}, \mathrm{h}$ & $0.22 \mathrm{~h}, \mathrm{i}$ & 0.27 & d,e \\
\hline $\mathrm{FCP}+\mathrm{WD} 5^{\circ} \mathrm{C}, 1 \mathrm{~min}$ & $0.82 \mathrm{a}$ & $0.45 \mathrm{c}$ & $0.21 \mathrm{~h}, \mathrm{i}$ & 0.50 & $\mathbf{a}$ \\
\hline $\mathrm{FCP}+\mathrm{WD} 50^{\circ} \mathrm{C}, 1 \mathrm{~min}$ & $0.17 \mathrm{~h}, \mathrm{i}$ & $0.18 \mathrm{~h}, \mathrm{i}$ & $0.16 \mathrm{i}$ & 0.17 & e \\
\hline $\mathrm{FCP}+\mathrm{WD} 50^{\circ} \mathrm{C}, 3 \mathrm{~min}$ & $0.65 \mathrm{~b}$ & $0.32 \mathrm{e}, \mathrm{f}, \mathrm{g}$ & $0.24 \mathrm{~g}, \mathrm{~h}, \mathrm{i}$ & 0.40 & b, c \\
\hline Mean & $0.48 \mathrm{~A}$ & 0.31 B & $0.23 \mathrm{C}$ & & \\
\hline
\end{tabular}

Significance

$$
\begin{array}{rr}
\text { Treatment }(\mathrm{T}) & * * * * \\
\text { Storage }(\mathrm{t}) & * * * * \\
\mathrm{~T} \times \mathrm{t} & * * * *
\end{array}
$$

Capital letters compare means along storage, while small letters compare means among treatment. Means not connected by same letter are significantly different according to Tuckey's test at $P<0.05$. n.d.: not determined, NS: not significant, $* P<0.05$, $* * P<0.01, * * * P<0.001, * * * * P<0.0001$.

\begin{tabular}{|c|c|c|c|c|}
\hline \multirow{2}{*}{ Treatment } & \multicolumn{3}{|c|}{ Storage (d) } & \multirow{2}{*}{ Mean } \\
\hline & 1 & 3 & 6 & \\
\hline $\mathrm{WD} 5^{\circ} \mathrm{C} 1 \mathrm{~min}+\mathrm{FCP}$ & n.d & n.d & n.d & n.d. \\
\hline $\mathrm{WD} 50^{\circ} \mathrm{C} 3 \mathrm{~min}+\mathrm{FCP}$ & $0.54 \mathrm{e}$ & $0.42 \mathrm{f}, \mathrm{g}$ & $0.50 \mathrm{e}, \mathrm{f}$ & 0.49 b \\
\hline $\mathrm{WD} 50^{\circ} \mathrm{C} 6 \mathrm{~min}+\mathrm{FCP}$ & $0.42 \mathrm{f}, \mathrm{g}$ & $0.40 \mathrm{~g}, \mathrm{~h}$ & $0.24 \mathrm{j}, \mathrm{k}$ & 0.35 c \\
\hline $\mathrm{FCP}+\mathrm{WD} 5^{\circ} \mathrm{C}, 1 \mathrm{~min}$ & $0.20 \mathrm{k}, 1$ & $0.31 \mathrm{j}$ & $0.30 \mathrm{j}$ & $0.27 \mathrm{~d}$ \\
\hline $\mathrm{FCP}+\mathrm{WD} 50^{\circ} \mathrm{C}, 1 \mathrm{~min}$ & $0.70 \mathrm{~d}$ & $0.94 \mathrm{~b}$ & $1.24 \mathrm{a}$ & 0.96 a \\
\hline $\mathrm{FCP}+\mathrm{WD} 50^{\circ} \mathrm{C}, 3 \mathrm{~min}$ & $0.79 \mathrm{c}$ & $0.42 \mathrm{f}, \mathrm{g}$ & $0.17 \quad 1$ & 0.48 b \\
\hline Mean & $0.52 \mathrm{~A}$ & 0.49 B & 0.49 В & \\
\hline
\end{tabular}

Table 7. Antioxidant activity $\left(\mathrm{g}_{\mathrm{fg}} \cdot \mathrm{mg}_{\mathrm{DPPH}}{ }^{-1}\right)$ of MP 'Ryan Sun' peaches submitted to hot water dips before or after processing and stored $6 \mathrm{~d}$ at $5{ }^{\circ} \mathrm{C}$.

Significance

$$
\begin{array}{rc}
\text { Treatment }(\mathrm{T}) & * * * * \\
\text { Storage }(\mathrm{t}) & * * \\
\mathrm{~T} \times \mathrm{t} & * * * *
\end{array}
$$

Capital letters compare means along storage, while small letters compare means among treatment. Means not connected by same letter are significantly different according to Tuckey's test at $P<0.05$. n.d.: not determined, NS: not significant, $* P<0.05$, $* * P<0.01$, *** $P<0.001$, **** $P<0.0001$.

sweetness $(p<0.0001)$, flesh browning $(p<0.01)$ and flavor $(p<0.0001)$. Aroma was reduced from 7.82 to 4.83 , sweetness from 8.61 to 7.40 , browning from 8.58 to 6.92 , taste from 8.04 to 5.95 and acceptability from 5.64 to 4.62 (Table 8 ).

In the principal components (PC) analysis, the three first PC's explained $65 \%$ of the variability. The first PC (32\%) was defined by sweetness and flavor (taste parameters). PC2 was defined by aroma (negative orientation), texture, acceptability and browning (positive orientation) and explained $20 \%$ of variability (Fig. 2 B). PC3 was responsible for $13 \%$ of the variability and was defined by sourness (data not shown). This analysis allowed the separation of treatments T1 (whole fruit submitted $1 \mathrm{~min}$ to water dip at $5{ }^{\circ} \mathrm{C}$ before fresh-cut processing), $\mathrm{T} 2$ (whole fruit submitted $3 \mathrm{~min}$ to hot water dip at $50^{\circ} \mathrm{C}$ before fresh-cut processing), $\mathrm{T} 3$ (whole fruit submitted 6 min to hot water dip at $50{ }^{\circ} \mathrm{C}$ before fresh-cut 
Table 8. Results of the sensory evaluation of fresh cut 'Ryan Sun' peaches submitted to hot water dips before or after processing and stored $6 \mathrm{~d}$ at $5{ }^{\circ} \mathrm{C}$.

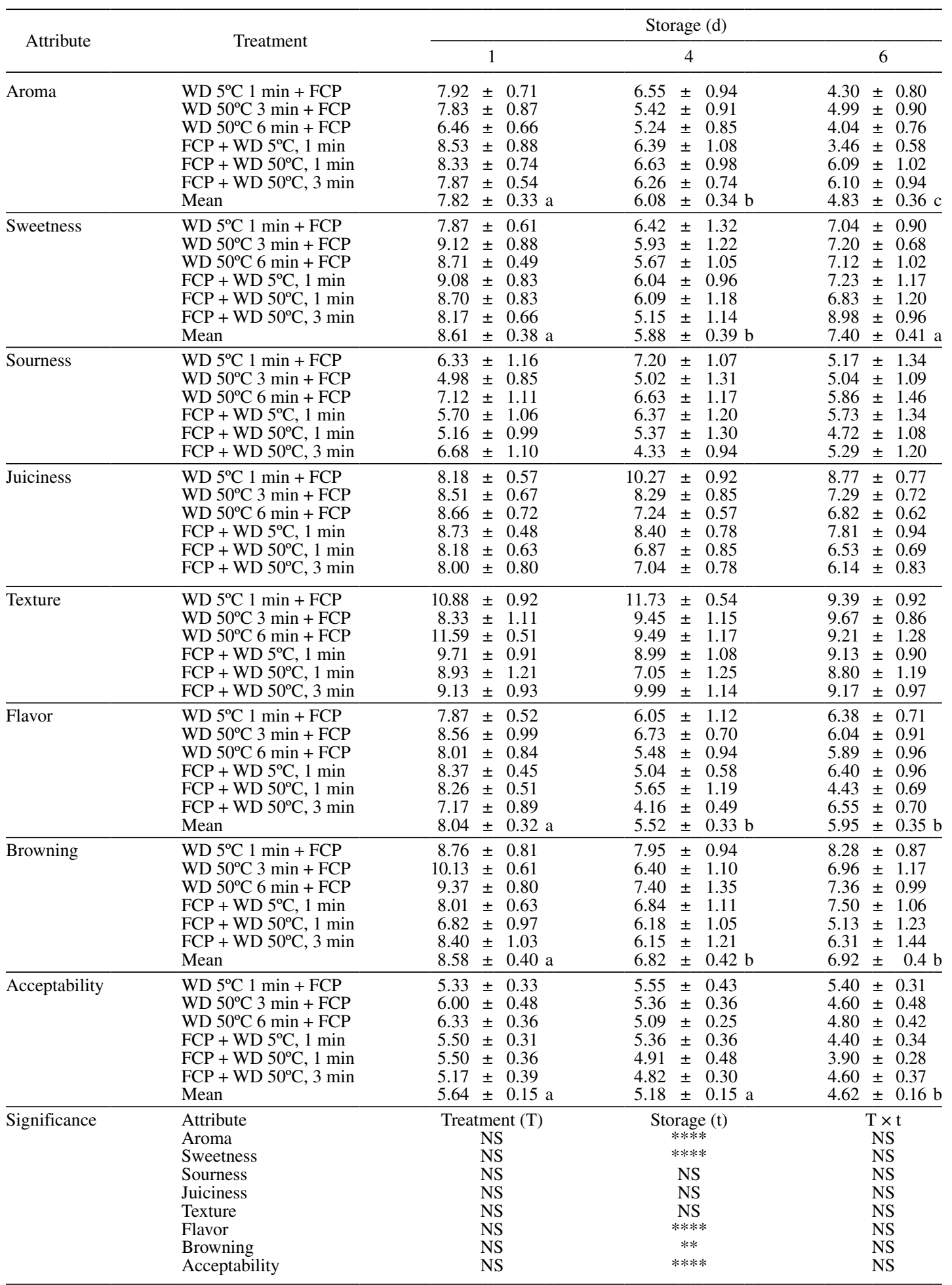

Means not connected by same letter are significantly different according to Tuckey's test at $p<0.05$. NS: not significant, $* P<0.05$, ** $P<0.01, * * * P<0.001$, **** $P<0.0001$. 
processing), T4 (fresh-cut peaches submitted 1 min to water dip at $5{ }^{\circ} \mathrm{C}$ ) and T5 (fresh-cut peaches submitted $1 \mathrm{~min}$ to hot water dip at 50 ${ }^{\circ} \mathrm{C}$ ) on day 1 from the other treatments evaluated throughout storage (Fig. 2A). The analysis of these attributes also revealed a moderate interaction $(r=0.5)$ between flavor versus sweetness, and flavor versus acceptability (Table 9).
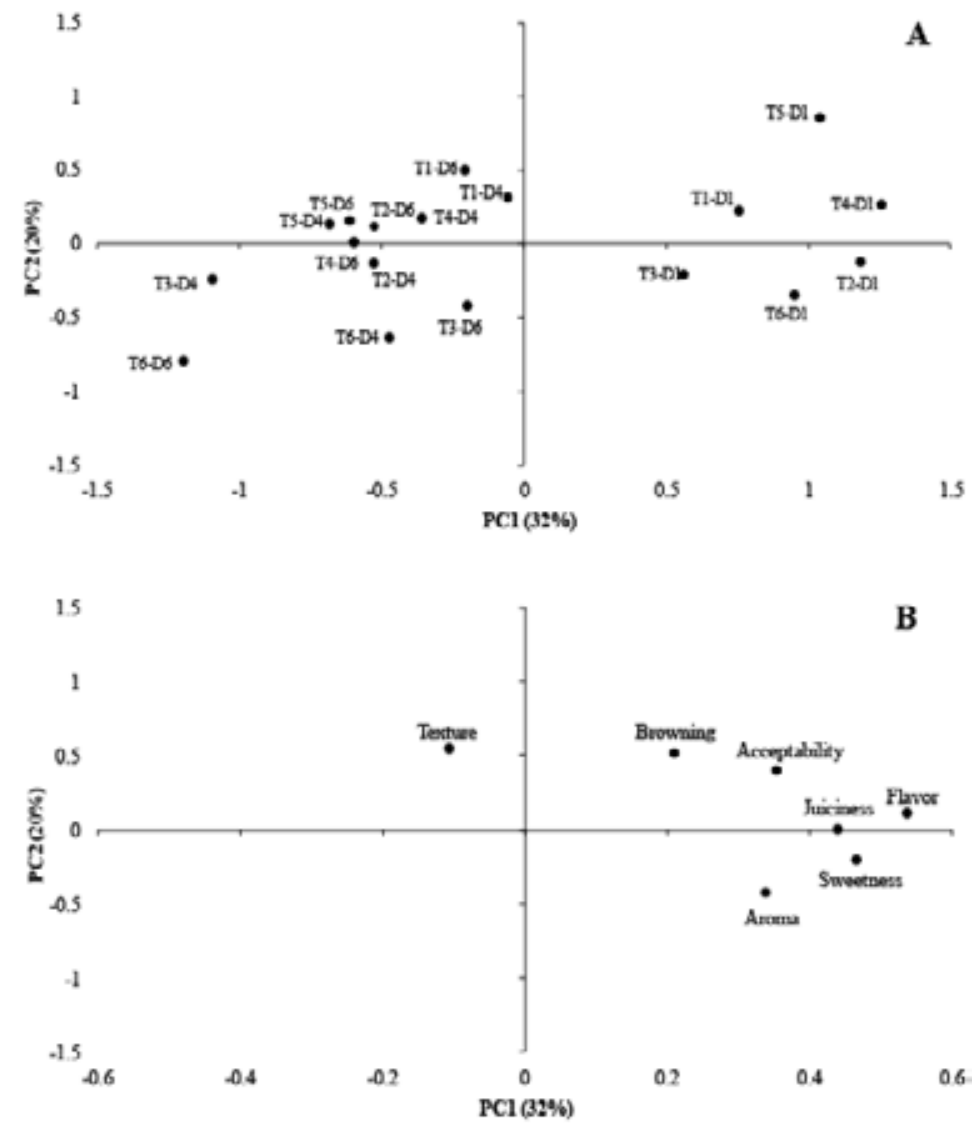

Fig. 2. Plot of centroid loadings (A) and scores (B) of principal components analysis of the sensory evaluation data of fresh-cut Ryan Sun peaches submitted to hot water dip before or after processing and stored $6 \mathrm{~d}$ at $5{ }^{\circ} \mathrm{C}$. The first two PC's explained $32 \%$ and $30 \%$ of total variance, respectively. Only eigenvectors $>0.20$ of the sensory evaluation data were represented in the loading plot. T1 corresponds to the whole fruit submitted to hot water dip 1 min at $5{ }^{\circ} \mathrm{C}$ before fresh-cut processing; $\mathrm{T} 2$ and $\mathrm{T} 3$ are the whole fruit submitted to hot water $\operatorname{dip} 1 \mathrm{~min}$ at $5{ }^{\circ} \mathrm{C}$ before fresh-cut processing, respectively; $\mathrm{T} 4$ represents the fresh-cut peaches submitted $1 \mathrm{~min}$ to water dip at $5{ }^{\circ} \mathrm{C}$; T5 and T6 are the fresh-cut peaches submitted 1 or 3 min to water dip at $50{ }^{\circ} \mathrm{C}$. D 1,4 and 6 are the storage days.

Table 9. Pearson product moment correlation coefficients found in the principal components analysis (PCA) for sensory analysis data of fresh cut 'Ryan Sun' peaches submitted to hot water dips before or after processing and stored $6 \mathrm{~d}$ at $5{ }^{\circ} \mathrm{C}$.

\begin{tabular}{|c|c|c|c|c|c|c|c|c|}
\hline & Aroma & Sweetness & Sourness & Juiciness & $\begin{array}{l}\text { Tex } \\
\text { ture }\end{array}$ & Flavor & Browning & Acceptability \\
\hline Aroma & 1 & & & & & & & \\
\hline Sweetness & 0.44 & 1 & & & & & & \\
\hline Sourness & 0.12 & 0.08 & 1 & & & & & \\
\hline Juiciness & 0.24 & 0.36 & 0.17 & 1 & & & & \\
\hline Texture & -0.30 & -0.15 & -0.05 & -0.11 & 1 & & & \\
\hline Flavor & 0.31 & 0.59 & -0.06 & 0.48 & -0.05 & 1 & & \\
\hline Browning & -0.03 & 0.07 & -0.10 & 0.18 & 0.23 & 0.26 & 1 & \\
\hline Acceptability & 0.01 & 0.15 & 0.10 & 0.30 & 0.12 & 0.50 & 0.33 & 1 \\
\hline
\end{tabular}




\section{Discussion}

Ryan Sun peaches are characterized as sweet, with a nearly full red blush over a cream-yellow background red skin color. Their flesh is yellow, flavorful, sweet and firm, which makes this cultivar excellent for slicing and eating. Baldwin et al. (2002) considered that repeat purchases of fruit and vegetables are determined by mouth-feel and flavor, but appearance and firmness determined the first impression of consumers. The characteristics of Ryan Sun peaches at harvest used in this study, mainly firmness, are within the parameter ranges established for the optimum maturity stage before processing. Gorny et al. (1998) proposed an optimum firmness range for Ryan Sun peaches destined to be fresh-cut between 2.9- $4.9 \mathrm{kgf}$ in order to resist quality degradation due to processing operations. In addition, the total phenol content identified in Ryan Sun peaches at harvest was similar to the content reported for Spring Belle peaches at harvest (Koukounaras et al., 2008). However, Wu et al. (2004) reported that peach, pineapple and watermelon have low total phenol content and antioxidant capacity (around 3-5 fold lower) compared to blueberry, raspberry and strawberry.

The quality of the fresh-cut product is altered by wounding (e.g. cutting, slicing or dicing), which increases respiration rate and causes major tissue disruption that is accompanied by flavor loss, cut surface discoloration, color loss, decay, increased rate of vitamin loss, rapid softening, shrinkage and shorter storage-life (Siddiq et al., 2013; Toivonen and DeEll, 2002; Watada and Qi, 1999; Wiley, 1994). In this study all fresh-cut treatments had a respiration rate approximately $24 \%$ greater than the whole fruit $\left(21.5 \mathrm{mg} \mathrm{CO} \cdot \mathrm{kg}^{-1} \cdot \mathrm{h}^{-1}\right)$ at the beginning of storage; it reached $15 \%$ greater $\left(34.05 \mathrm{mg} \mathrm{CO} \cdot \mathrm{kg}^{-1} \cdot \mathrm{h}^{-1}\right)$ at the end of storage $(6 \mathrm{~d})$ at $5^{\circ} \mathrm{C}$. Similar results have been reported in fresh-cut Keitt mangoes subjected to hot water dipping at 46 or $50^{\circ} \mathrm{C}$ for 30 or $75 \mathrm{~min}$, cooled for $15 \mathrm{~min}$, minimally processed and stored at $6^{\circ} \mathrm{C}$ for $9 \mathrm{~d}$ (Djioua et al., 2009).

One of the parameters of quality which is altered in fresh-cut products due to wounding and the physiological behavior is texture. Tissue softening is a very serious problem with fresh-cut fruit products, which can limit shelf-life. Consumers expect crispy fresh-cut fruits and those soft fruits, such as cantaloupe and peach, should yield to chewing without being mushy (Beaulieu, 2011; Barrett et al.,
2010; Beaulieu and Gorny, 2004). Fresh-cut fruit firmness is an important quality attribute that can be affected by cell softening enzymes present in the fruit tissue and by decreased turgor due to water loss (Beaulieu and Gorny, 2004). The low firmness loss observed in fresh-cut peaches submitted to water dip $3 \mathrm{~min}$ at $50{ }^{\circ} \mathrm{C}$ may be explained by pectin methyl esterase deactivation, which releases the methoxyl groups from galacturonic acid residues of pectic substances, which are thus available for complexing free cations, particularly endogenous calcium $\left(\mathrm{Ca}^{2+}\right)$ forming Ca-pectates, increasing rigidity of the middle lamella and cell wall (Abreu et al., 2003).

An important issue in fresh-cut fruit processing is the control of discoloration (pinking, reddening or blackening) or browning of cut surfaces (Adams and Brown, 2007; Beaulieu, 2011; Beaulieu and Gorny, 2004). Color is derived from the natural pigments in fruits and vegetables, many of which change as the plant proceeds through maturation and ripening; color attracts the consumer to a product and can help in impulse purchases. For this reason fresh-cut fruits must appear to be fresh, generally indicated by the brightness of color (Barrett et al, 2010). The hot water dips were more effective in retarding the loss of lightness $\left(\mathrm{L}^{*}\right)$ of the flesh color in Ryan Sun peaches treated before fresh-cut processing than fruit submitted to fresh-cut processing before hot water dip at $50^{\circ} \mathrm{C}$. Kim et al. (1993) found that Delicious, Empire, Golden Delicious, McIntosh and New York 674 apple slices prepared from heat-treated fruit at $45^{\circ} \mathrm{C}$ for $105 \mathrm{~min}$ did not display browning after treatment, compared to those prepared from non-treated apples. Loaiza-Velarde and Saltveit (2001) also observed that the maximum reduction in browning in fresh cut lettuce occurred in lettuce heat-treated around $6 \mathrm{~h}$ before cutting. On the other hand, Abreu et al. (2003) found that fresh-cut Rocha pears submitted to mild heat treatments (between 34 and $\left.45^{\circ} \mathrm{C}\right)$ were more effective $(p</ 0.01)$ in preserving lightness $\left(\mathrm{L}^{*}\right)$ regardless of treatment time, but temperatures over $45^{\circ} \mathrm{C}$ enhanced cut surface discoloration, which could increase tissue damage associated with cell death.

Consumer acceptance of fresh-cut fruits most often relies upon the inherent flavor and texture quality of the product, so improving consistency in fresh-cut fruit product flavor and texture may enhance continuous consumer purchase of these products (Beaulieu, 2011; Beaulieu and Gorny, 2004). To 
achieve this, fresh-cut fruit must be sweet without the presence of off-flavors. Because sweetness increases with ripening and ripe fruits deteriorate more rapidly, most fruits must be harvested before full sweetness is achieved (Barrett et al., 2010). The change in flavor attributes (SSC, $\mathrm{pH}$, but mainly in TA) may be related to the high physiological activity identified in fresh-cut peaches in comparison to intact fruit (Fig. 1). The soluble solid content (SSC) is generally positively correlated with desirable flavor quality in the food industry (Beaulieu and Gorny, 2004). In this study the SSC in fresh-cut peaches was not affected by hot water treatment or storage. Fan et al. (2008) reported that fresh-cut cantaloupe cubes prepared from hot water-treated cantaloupe fruit $\left(76^{\circ} \mathrm{C}\right.$ for $3 \mathrm{~min}$ ) had no consistent changes in soluble solids. $\mathrm{pH}$ has been reported to increase over time (Wright and Kader, 1997), but it cannot be used as an indicator of the quality because $\mathrm{pH}$ does not change significantly from amounts present in the freshly cut fruit, as was reported in fresh-cut Kent mango slices stored 2 weeks at $4{ }^{\circ} \mathrm{C}$ (Dea et al., 2010). However, the decrease in TA (as reported for fresh-cut Ryan Sun peaches here) could be used as a good quality parameter in fresh-cut peaches, since it represents a clear relationship between the metabolism of organic acids as respiratory substrates and taste, as has also been found in other fruit (Budde et al., 2006; Kim et al., 1993). In addition, TA and SSC have also been used to assess quality via the SSC:TA ratio in some fresh-cut fruit (Beaulieu and Gorny, 2004) as a measure of the organic acid and sweetness balance, which defines flavor and influences consumer perception. In the present study, hot water dip at $50^{\circ} \mathrm{C}$ applied to whole Ryan Sun peaches before fresh-cut processing were more effective to preserve TA.

Consumers also expect fresh fruits and vegetables to be good sources of dietary fiber and functional compounds, such as total phenols and antioxidant capacity. Unfortunately, they have no way of distinguishing between individual products that have high versus low content of nutrients (Beaulieu, 2011; Barrett et al., 2010). Phenol compounds are involved in lignin synthesis and the strengthening of cell walls, and they are also of major importance for their contribution to flavor, stability and nutritional value of fruits and vegetables (Campos-Vargas and Saltveit, 2002). In this study all treatments produced an increase in the total phenol content in comparison to raw material at harvest as a response to the stress produced by cutting, as has been reported previously (Heredia and Cisneros, 2007; Vicente et al., 2003). The total phenol content observed in this study at the end of storage might be also related to the inactivation of polyphenol oxidase (PPO), responsible for browning (Adams and Brown, 2007; Queiroz et al., 2008), by the treatments applied to Ryan Sun peaches.

The sensory evaluation revealed that sweetness and taste were more affected by storage. However, these parameters did not affect the acceptability by judges, since they showed a weak correlation with acceptability ( $r=0.15$ and 0.10 , for sweetness and sourness, respectively). The acceptability of fresh cut Ryan Sun peaches by judges might be due to taste, which is defined by the sensations of taste, smell, and pressure in the mouth (Beaulieu, 2011; Barnett et al., 2010) as was revealed by a moderate correlation $(r=0.50)$. In this study, the taste of fresh-cut Ryan Sun peaches might be influenced by the TA change, which was reduced from $0.48 \%$ to $0.40 \%$, even though whole peaches previously dipped $6 \mathrm{~min}$ in hot water at $50^{\circ} \mathrm{C}$ and then submitted to fresh cut processing showed higher TA than peaches dipped $3 \mathrm{~min}$ in hot water at $50{ }^{\circ} \mathrm{C}$ after processing.

\section{Conclusions}

The results of this study demonstrate the efficiency of hot water dip at $50{ }^{\circ} \mathrm{C}$ for $3 \mathrm{~min}$ before fresh-cut processing on Ryan Sun peaches for maintaining the firmness, lightness and hue angle of the flesh color. This is probably an effect of the inactivation of enzymes related to browning and softening. In addition, this treatment allowed the fresh-cut peaches to have the highest TA, which positively influenced product flavor and thus consumer acceptability. The total phenol content and antioxidant capacity measured in Ryan Sun peaches submitted to $3 \mathrm{~min}$ hot water dip at $50{ }^{\circ} \mathrm{C}$ before fresh-cut processing appears to have inactivated the enzymes related to the oxidation of these compounds. Thus this treatment is good tool to provide consumers a product with good functional quality.

\section{Acknowledgements}

This study was funded by CONICYTFONDEF n ${ }^{\circ}$ D07I1026 (Chile). J.M. Obando-Ulloa 
was supported by CONICYT- PROGRAMA DE INSERCIÓN DE CAPITAL HUMANO AVANZADO A LA ACADEMIA n ${ }^{\circ} 791100025$ and CONICYT-FONDECYT n ${ }^{\circ} 3100074$ (Chile). We thank Daniela Cárdenas and Mariela Labbé for technical assistance.

\section{Literature Cited}

Abreu, M.; Beirão-da-Costa, S.; Gonçalves, E.M.; Beirão-daCosta, M.L.; Moldão-Martins, M.

2003. Use of mild heat pre-treatments for quality retention of fresh-cut 'Rocha' pear. Postharvest Biology and Technology, 30: $153-160$.

Adams, J.B.; Brown, H.M.

2007. Discoloration in raw and processed fruits and vegetables. Critical Reviews in Food Science and Nutrition, 47: 319-333.

Baldwin, E.A.

2002. Fruit flavor, volatile metabolism and consumer perceptions. In: Knee, M. (ed.), Fruit quality and its biological basis. CRC Press, LLC., Boca Raton, FL, United States of America. p. 89-106.

Barrett, D.M.; Beaulieu, J.C.; Shewfel, R.

2010. Color, flavor, texture, and nutritional quality of freshcut fruits and vegetables: Desirable levels, instrumental and sensory measurement, and the effects of processing. Critical Reviews in Food Science and Nutrition, 50: 369-389.

Beaulieu. J.C.

2011. Factors affecting sensory quality of fresh-cut produce. In: Martín-Belloso O. and R. Soliva-Fortuny (eds.), Advances in Fresh-Cut Fruits and Vegetables Processing. CRC Press, Taylor and Francis, Boca Raton, FL., United States of America. p. 115-143.

Beaulieu, J.C., Ingber, B.F.; Lea, J.M.

2011. Physiological, volatile, and SEM surface effects resulting from cutting and dipping treatments in cantaloupe. Journal of Food Science, 76: 415-422.

Beaulieu J.C., Gorny, J.R.

2004. Fresh-cut Fruits. p. 17. In: Gross, KC, C.Y. Wang, and M.E. Saltveit (eds.), USDA Handbook $\mathrm{N}^{\circ}$ 66. The commercial storage of fruits, vegetables, and florist and nursery stocks. $3^{\text {rd }}$ edition, Washington D.C.: Agricultural Research Service. Available at http://www.ba.ars.usda.gov/ hb66/146freshcutfruits.pdf. Accessed: September 2014.

Brand-Williams, W., Cuvelier, M.; Berset, C.

1995. Use of a free radical method to evaluate antioxidant activity. LWT- Food Science and Technology, 28: 25-30.

Budde, C.O.; Polenta, G.; Lucangeli, C.D.; Murray, R.E.

2006. Air and immersion heat treatments affect ethylene production and organoleptic quality of 'Dixiland' peaches. Postharvest Biology and Technology, 41: 32-37.

Campos-Vargas, R.; Saltveit, M.E.

2002. Involvement of putative chemical wound signals in the induction of phenolic metabolism in wounded lettuce. Physiologia Plantarum, 114: 73-84.

Dea, S., Brecht, J.K.; Nunes, M.C.N.; Baldwin, E.A.

2010. Quality of fresh-cut 'Kent' mango slices prepared from hot water or non-hot water-treated fruit. Postharvest Biology and Technology, 56: 171-180.

Djioua, T.; Charles, F.; Lopez-Lauri, F.; Filgueiras, H.; Coudret,

A.; Freire Jr., M.; Ducamp-Collind, M.N.; Sallanon, H.

2009. Improving the storage of minimally processed mangoes (Mangifera indica L.) by hot water treatments. Postharvest Biology and Technology, 52: 221-226.
Fan, S.; Bielenberg, D.G.; Zhebentyayeva, T.N.; Reighard, G.L.; Okie, W.R.; Holland, D.; Abbott, A.G.

2010. Mapping quantitative trait loci associated with chilling requirement, heat requirement and bloom date in peach (Prunus persica). New Phytologist, 185: 917-930.

Fan, X.; Annous, B.A.; Beaulieu, J.C.; Sites, J.

2008. Effect of hot water surface pasteurization of whole fruit on shelf-life and quality of fresh-cut cantaloupe. Journal of Food Science, 73: 91-98.

Fernández-Trujillo, J.P.; Artés, F.

1997. Keeping quality of cold stored peaches using intermittent warming. Food Research International, 30: 441-450.

Fernández-Trujillo, J.P.; Obando, J.; Martínez, J.A.; Alarcón,

A.L.; Eduardo, I.; Arús, P.; Monforte. A.J.

2005. In. Atienza, J., and J. Rabasseda (eds.). Quality management of experiments with a collection of nearisogenic lines of melon. In Proceedings of the Third Virtual Iberoamerican Conference on Laboratory Quality Management. III IBEROLAB. Madrid, Spain. pp. 149-158.

Heredia, B.; Cisneros, L.

2009. The effect of exogenous ethylene and methy jasmonate on PAL activity, phenolic profiles and antioxidant capacity of carrots (Daucus carota L.) under different wounding intensities. Postharvest Biology and Technology, 51: 242-249.

Kang, H.M.; Saltveit, M.E.

2001. Activity of enzymatic antioxidant defense systems in chilled and heat shocked cucumber seedling radicles. Physiologia Plantarum, 113: 548-556.

Kim, D.M.; Smith, N.L.; Lee, C.Y.

1993. Apple cultivar variations in response to heat treatment and minimal processing. Journal of Food Science, 58: 1111-1124.

Koukounaras, A.; Diamantidis, G.; Sfakiotakis, E.

2008. The effect of heat treatment on quality retention of fresh-cut peach. Postharvest Biology and Technology, 48: 30-36.

Loaiza-Velarde, J.G.; Mangrich, M.E.; Campos-Vargas, R.;

Saltveit, M.E.

2003. Heat shock reduces browning of fresh-cut celery petioles. Postharvest Biology and Technology, 27: 305-311.

Loaiza-Velarde, J.G.; Saltveit, M.E.

2001. Heat shocks applied either before or after wounding reduced browning of lettuce leaf tissue. Journal of the American Society for Horticultural Science, 126: 227-234. Lurie, S.

2006. The effect of high temperature treatment on quality of fruits and vegetables. Acta Horticulturae, 712: 158-164.

McGuire, R.

1992. Reporting of objective color measurements. HortScience, 27: 1254-1255.

Obando, J.; Fernández-Trujillo, J.P.; Martínez, J.A.; Alarcón, A.L.; Eduardo, I.; Arús, P.; Monforte, A.J.

2008. Identification of melon fruit quality quantitative trait loci using near-isogenic lines. Journal of the American Society for Horticultural Science, 133: 139-151. 
Obando-Ulloa, J.M.; Moreno, E.; García-Mas, J.; Nicolai, B.; Lammertyn, J.; Monforte, A.J.; Fernández-Trujillo, J.P.

2008. Climacteric or non-climacteric behavior in melon fruit 1. Aroma volatiles. Postharvest Biology and Technology, 49: $27-37$

Oms-Oliu, G.; Rojas-Grau, M.A.; Alandes González, L.; Varela, P.; Soliva-Fortuny, R.; Hernando Hernando, M.I.; Perez Munuera, I.; Fiszman, S.; Martin-Belloso, O.

2010. Recent approaches using chemical treatments to preserve quality of fresh-cut fruit: A review. Postharvest Biology and Technology, 57: 139-148.

Queiroz, C.; Mendes Lopes, M.L.; Fialho, E.; Valente-Mesquita, V.L.

2008. Polyphenol oxidase: Characteristics and mechanisms of browning control. Food Reviews International, 24: 361-375.

Rodríguez, S.; Casóliba, R.M.; Questa, A.G.; Felker, P. 2005. Hot water treatment to reduce chilling injury and fungal development and improve visual quality of two Opuntia ficus indica fruit clones. Journal of Arid Environments, 63: 366-378.

Saltveit, M.

2005. Influence of heat shocks on the kinetics of chillinginduced ion leakage from tomato pericarp discs. Postharvest Biology and Technology, 36: 87-92.

Saltveit, M.

2001. Chilling injury is reduced in cucumber and rice seedlings and in tomato pericarp discs by heat-shocks applied after chilling. Postharvest Biology and Technology, 21: 169-177.

Saltveit, M.

2000. Wound induced changes in phenolic metabolism and tissue browning are altered by heat shock. Postharvest Biology and Technology, 21: 61-69.

Siddiq, M., D.S. Sogi, and K.D. Dolan.

2013. Antioxidant properties, total phenolics, and quality of fresh-cut 'Tommy Atkins' mangoes as affected by different pre-treatments. LWT-Food Science and Technology, 53: 156-162.

Steiner, A.; Abreu, M.; Correira, L.; Beirao-da-Costa, S.; Leitao, E.; Beirao-da-Costa, M.; Empis, J.; Moldao-Martins, M.

2006. Metabolic response to combined mild heat pre-treatments and modified atmosphere packaging on fresh-cut peach. European Food Research and Technology, 22: 217-222.

Toivonen, P.M.A.; DeEll, J.R.

2002. Physiology of Fresh-cut Fruits and Vegetables. In: Lamikanra, O (ed.), Fresh-Cut Fruits and Vegetables. Science, Technology and Market. Boca Raton, FL: CRC Press LLC, pp. 91-123.

Velioglu, Y.; Mazza, G.; Gao, L.; Oomah, B.

1998. Antioxidant activity and total phenolics in selected fruits, vegetables, and grain products. Journal of Agriculture and Food Chemistry, 46: 4113-4117.

Vicente, A.R.; Martínez, G.A.; Chaves, A.R.; Civello, P.M.

2003. Influence of self-produced $\mathrm{CO}_{2}$ on postharvest life of heat-treated strawberries. Postharvest Biology and Technology, 27: 265-275.

Watada, A.E.; Qi, L.

1999. Quality of fresh-cut produce. Postharvest Biology and Technology, 15: 201-205.

Wiley R.C.

1994. Minimally processed refrigerated fruits and vegetables, London, UK: Chapman \& Hall. 362 pp.

Wright, K.P.; Kader, A.A.

1997. Effect of slicing and controlled-atmosphere storage on the ascorbate content and quality of strawberries and persimmons. Postharvest Biology and Technology, 10: 39-48.

Wu, X.; Beecher, G.; Holden, J.; Haytowitz, D.; Gebhardt, S.; Prior, R.

2004. Lipophilic and hydrophilic antioxidant capacities of common foods on the United States. Journal of Agriculture and Food Chemistry, 52: 4026-4037. 